\title{
Haste intramedular bloqueada em comparação com placa de compressão dinâmica na estabilização de osteotomia intertrocantérica varizante em cães: estudo ex vivo ${ }^{1}$
}

\author{
Leandro Z. Carneiro ${ }^{2}$, Maria L.A. Mistieri' ${ }^{2 *}$, Carlos R. Cauduro ${ }^{3}$, Alisson F. Rosa ${ }^{3}$ \\ e João P.E. Pascon ${ }^{2}$
}

\begin{abstract}
Carneiro L.Z., Mistieri M.L.A., Cauduro C.R., Rosa A.F. \& Pascon J.P.E. 2017. [Interlocking nail system in comparison with dynamic compression plate in the stabilization of intertrochanteric varus osteotomy in dogs: study in cadavers.] Haste intramedular bloqueada em comparação com placa de compressão dinâmica na estabilização de osteotomia intertrocantérica varizante em cães: estudo ex vivo. Pesquisa Veterinária Brasileira 37(12):1474-1478. Hospital Universitário Veterinário, Universidade Federal do Pampa, BR-472 Km 592, Uruguaiana, RS 97508-000, Brazil. E-mail: malimistieri@gmail.com

Considering the advantages of the interlocking intramedullary nail (IN) in relation to bone plates in the femoral stabilization, this study aimed to evaluate the feasibility and effectiveness of IN application post intertrochanteric varus osteotomy (IVO). For this purpose, 10 canines cadavers were used. On the left femurs, the fixation was obtained with IN (IN group) and rights fixed with dynamic compression plates and screws (plate group). Was compared the angles of Norberg and inclination of the head and femoral neck (IHF) before (T0) and after (T1) IVO. The results of time spent for placement of implants and biomechanical resistance to compression and torsion were also confronted between groups. There was an increase of the Norberg angle between T0 $\left(106.84 \pm 5.55^{\circ}\right)$ and T1 $\left(111.22^{\circ} \pm 3.89\right)$, only in IN group $(\mathrm{p}<0.05)$. However, reduction of IHF angle after IVO (T1) was observed in both, the plate group ( $\mathrm{T} 0=127.6 \pm 4.70^{\circ}$ e $\left.\mathrm{T} 1=110.06 \pm 10.61^{\circ}, \mathrm{p}<0.05\right)$ and IN group $\left(\mathrm{T} 0=126.43 \pm 5.87^{\circ}\right.$ e $\left.\mathrm{T} 1=116.87 \pm 8.62^{\circ}, \mathrm{p}<0.05\right)$. The placement times of the implants did not differ statistically and only the compression biomechanical test revealed differences between groups, with greater resistance $(\mathrm{P}=0.033)$ of the plate group $(863.3 \pm 74.46 \mathrm{~N} / \mathrm{mm})$ compared to IN group $(586.7 \pm 44.10 \mathrm{~N} / \mathrm{mm})$. Thus, stabilization through IN was feasible and effective. Although the compression biomechanical results has demonstrated a higher fragility of IN in relation to the compression plate, their values are above the forces, reported in literature, acting in normal dog gait.
\end{abstract}

INDEX TERMS: Interlocking nail system, compression plate, stabilization, dogs, orthopedics, hip dysplasia, implants, intertrochanteric varus osteotomy.

RESUMO.- Tendo em vista as vantagens das hastes intramedulares bloqueadas (HIB) em relação às placas ósseas na estabilização femoral, o presente estudo objetivou avaliar a exequibilidade e eficácia da aplicação da HIB pós osteotomia intertrocantérica varizante (OIV). Submeteu-

\footnotetext{
${ }^{1}$ Recebido em 27 de outubro de 2015.

Aceito para publicação em 5 de abril de 2017.

${ }^{2}$ Hospital Universitário Veterinário (HUVet), Universidade Federal do Pampa (Unipampa), BR-472 Km 592, Uruguaiana, RS 97508-000, Brasil. *Autor para correspondência: malimistieri@gmail.com

${ }^{3}$ Colégio Politécnico, Universidade Federal de Santa Maria (UFSM), Av. Roraima 1000, Cx. Postal 5082, Cidade Universitária, Camobi, Santa Maria, RS 97105-900, Brasil.
}

-se 10 cadáveres caninos à OIV. Nos antímeros esquerdos obteve-se a estabilização por meio de HIB (grupo HIB) e, nos direitos, com placas de compressão dinâmica (grupo placa). Foram comparados os ângulos de Norberg e de inclinação da cabeça e colo femoral (ICF) antes (T0) e após (T1) a OIV. 0 tempo dispendido para a colocação dos implantes e resultados de resistência biomecânica à compressão e torção também foram confrontados entre os grupos. Houve aumento do ângulo de Norberg entre T0 $\left(106,84 \pm 5,55^{\circ}\right)$ e $\mathrm{T} 1\left(111,22^{\circ} \pm 3,89\right)$, apenas no grupo HIB $(\mathrm{p}<0,05)$. No entanto, redução do ângulo de ICF após OIV (T1) foi observada tanto no grupo placa (T0 $=127,6 \pm 4,70^{\circ}$ e $\left.\mathrm{T} 1=110,06 \pm 10,61^{\circ}, \mathrm{p}<0,05\right)$ quanto no grupo HIB 
( $\mathrm{T} 0=126,43 \pm 5,87^{\circ}$ e $\left.\mathrm{T} 1=116,87 \pm 8,62^{\circ}, \mathrm{p}<0,05\right)$. Os tempos de colocação dos implantes não diferiram estatisticamente e apenas o teste biomecânico de compressão revelou diferença entre os grupos, com maior resistência $(\mathrm{P}=0,033)$ do grupo placa $(863,3 \pm 74,46 \mathrm{~N} / \mathrm{mm})$ em relação ao grupo HIB $(586,7 \pm 44,10 \mathrm{~N} / \mathrm{mm})$. Deste modo, a estabilização por meio de HIB foi factível e eficaz. Embora o resultado biomecânico de compressão tenha demonstrado maior fragilidade da HIB em relação à placa de compressão, seus valores estão acima das forças atuantes, reportadas na literatura, na deambulação normal de cão.

TERMOS DE INDEXAÇ̃̃O: Haste intramedular bloqueada, placa de compressão dinâmica, estabilização, osteotomia intertrocantérica varizante, cães, displasia coxofemoral, implantes, biomecânico.

\section{INTRODUÇÃO}

Dentre as diversas alternativas de tratamento cirúrgico da displasia coxofemoral canina, a osteotomia intertrocantérica varizante (OIV) é indicada para animais jovens que apresentem ângulo de inclinação da cabeça e colo femoral (ICF) acima de $145^{\circ}$, (Pinna et al. 2013). A técnica reduz o ICF por meio da retirada de cunha óssea na epífise proximal do fêmur, a fim de melhorar a congruência articular (Evers et al. 1997).

A estabilização da OIV pode ser realizada por meio de diversos métodos, como pino intramedular, calha de Küntcher e placa com gancho, porém, a placa de compressão dinâmica é o mais usualmente aplicado (Inoe et al. 1996, Evers et al. 1997, Prieur 2005, Pinna et al. 2013). Sabe-se que a haste intramedular bloqueada (HIB) é capaz de bloquear as forças de compressão, rotação e flexão (Schmaedecke 2007). Além disso, apresenta superioridade biomecânica em relação à placa de compressão na estabilização de ossos longos, sendo também mais rapidamente implantada. Contudo, é um implante não moldável (Schmaedecke 2007, Piórek et al. 2012).

0 presente estudo objetivou verificar a exequibilidade e eficácia da aplicação da HIB em comparação à placa de compressão dinâmica na estabilização após OIV em cadáveres caninos.

\section{MATERIAL E MÉTODOS}

Foram utilizados 10 cadáveres caninos, livres de moléstia ortopédica que foram a óbito por causas não relacionadas ao estudo, totalizando 20 articulações coxofemorais saudáveis ao exame radiográfico. Destes, quatro eram fêmeas e seis machos, com peso superior a $15 \mathrm{~kg}$ (média 20,07士6,05 kg). Após constatado o óbito, os animais foram congelados a $-24^{\circ} \mathrm{C}$ por no máximo cinco dias e descongelados lentamente em geladeira $\left(8^{\circ} \mathrm{C}\right)$ por 24 horas antes do procedimento cirúrgico. 0 uso dos animais e a metodologia deste estudo foram aprovados pelo Comitê de Ética para o Uso de Animais (CEUA, protocolo 007/2012) da instituição de origem.

Em todos os fêmures foi realizada a técnica convencional de osteotomia intertrocantérica varizante (OIV) descrita por Evers et al. (1997). Com serra oscilatória pneumática e irrigação com solução fisiológica, fez-se primeiramente a osteotomia angulada em seguida a oblíqua entre os trocânteres maior e menor em ambos os fêmures de cada animal. Culminando na retirada de uma cunha óssea padronizada em 20 graus, com maior amplitude na porção medial. Nos antímeros direitos (grupo placa), os segmentos ósse- os foram reduzidos com uso de pinças ósseas espanholas promovendo discreta rotação medial do segmento proximal, objetivando à normoversão da cabeça femoral. A fixação se deu por meio de placa de compressão dinâmica de $3,5 \mathrm{~mm}$ com cinco orifícios (moldada no período transcirúrgico) e cinco parafusos de $3,5 \mathrm{~mm}$ de diâmetro. De modo similar em todos do grupo placa, a fixação teve início com a implantação do parafuso mais próximo à linha da osteotomia do segmento proximal, em seguida o parafuso contralateral à osteotomia foi posicionado de maneira compressiva, em ato contínuo o restante dos parafusos foi colocado de forma intercalada nos segmentos proximal e distal.

Nos fêmures esquerdos (grupo HIB), a inclinação do segmento proximal gerada após a redução dos segmentos não permitia o acesso da haste até o canal medular através da fossa intertrocantérica. Assim, utilizou-se broca de $4,5 \mathrm{~mm}$ para se obter acesso ao canal medular através do trocanter maior, posteriormente fresado com fresa manual de $6 \mathrm{~mm}$ de forma normógrada. A haste de $6 \mathrm{~mm}$, com quatro orifícios, foi introduzida também de forma normógrada com auxílio da régua guia e procedeu-se seu bloqueio com dois parafusos proximais e dois distais de $2,7 \mathrm{~mm}$ de diâmetro. Primeiramente o parafuso mais próximo da linha de osteotomia do segmento proximal foi fixado e em seguida o parafuso contralateral da osteotomia. Em ordem, os parafusos restantes foram implantados no segmento proximal e no segmento distal respectivamente. 0 comprimento das hastes e dos parafusos foi escolhido de acordo com a avaliação radiográfica de cada espécime. 0 porte dos animais selecionados neste estudo permitiu a padronização da espessura das hastes $(6 \mathrm{~mm})$ devido ao tamanho similar dos canais medulares avaliados radiograficamente. A escolha da HIB utilizada foi de acordo com Schmaedecke (2007), a qual deve preencher a maior parte do canal medular. Deste modo, as hastes de $6 \mathrm{~mm}$ possuem orifícios padrão para parafusos de 2,7 mm de diâmetro, impossibilitando a utilização de parafusos de maior diâmetro. 0 tempo da colocação de cada implante foi cronometrado.

Salienta-se que a régua guia e hastes utilizadas neste estudo, após estudo piloto, foram adaptadas pelo fabricante de forma a possuírem um centímetro entre a extremidade até o centro do orifício mais proximal da haste e entre seus orifícios proximais (Fig.1) para se adaptarem ao tamanho do segmento proximal.

Os animais foram submetidos à avaliação radiográfica das articulações coxofemorais imediatamente antes (T0) e após (T1) as técnicas cirúrgicas (Fig.2). Utilizou-se projeção ventrodorsal da pelve, como descrito por Ciarlini et al. (2009) e equipamento convencional de raios-x com sistema de leitura digital (Carestream Directview $\mathrm{CR}^{\circledR}$ ). Realizou-se a mensuração dos ângulos de Nor-

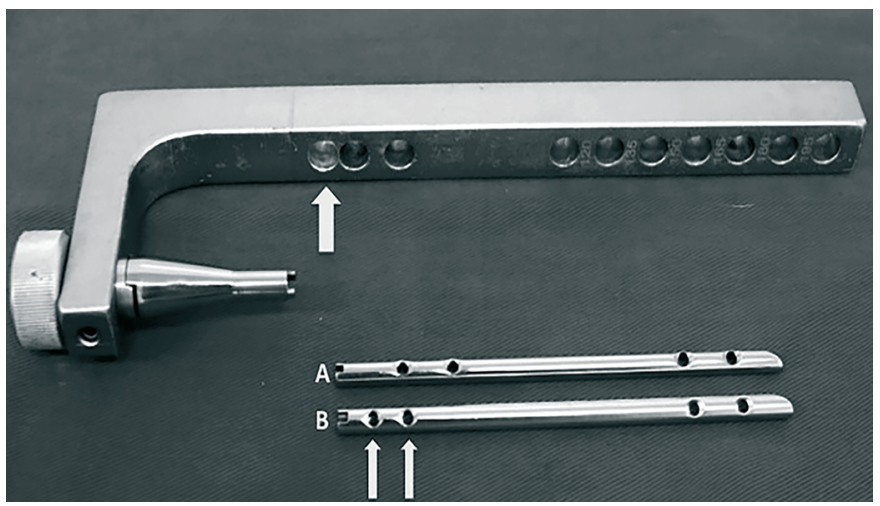

Fig.1. Adequações realizadas no kit de haste bloqueada. Novo orifício em régua guia (seta superior). Comparação entre os orifícios da haste convencional (A) com os orifícios (setas inferiores) da haste modificada (B). 


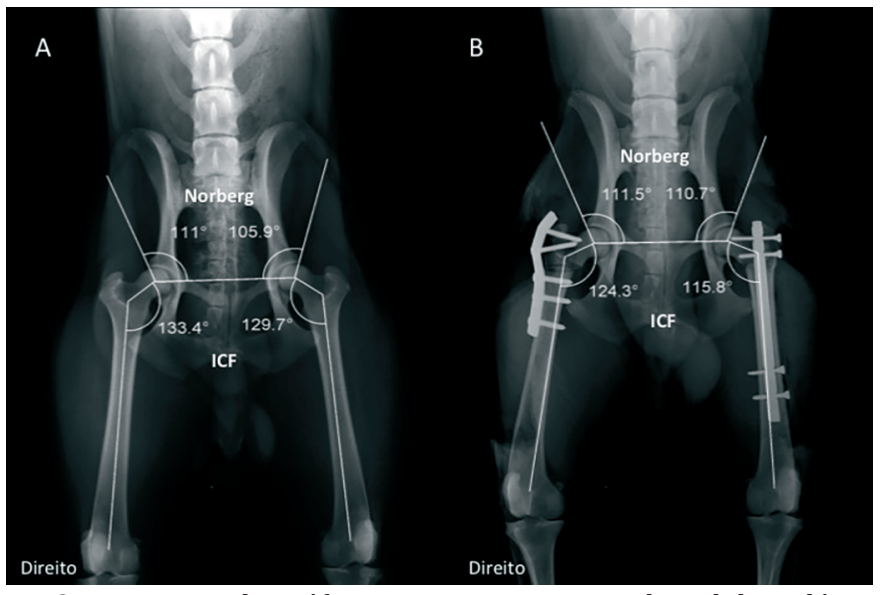

Fig.2. Imagens radiográficas na projeção ventrodorsal de cadáver de cão. Observar mensuração dos ângulos de Norberg e de inclinação de cabeça e colo femoral (ICF) antes (pré-cirúrgico (A) e após (pós-cirúrgico (B) a osteotomia intertrocantérica varizante (OIV). Na imagem B observa-se a colocação dos implantes; no fêmur direito placa compressiva e no esquerdo haste intramedular bloqueada (HIB).

berg de acordo com Vieira et al. (2010) e de inclinação de cabeça e colo femoral pelo método baseado no eixo de simetria realizado por Pinna et al. (2013). As mensurações foram efetuadas pelo mesmo avaliador, por meio de software (Directview $\mathrm{CR}^{\circledR}$ ).

Após exames radiográficos em T1, sete pares de fêmures, foram colhidos, embalados em sacos plásticos e criopreservados a $-24^{\circ} \mathrm{C}$ durante 30 dias. Para a realização dos testes biomecânicos destrutivos, os espécimes foram descongelados por oito horas até atingirem a temperatura ambiente, sendo frequentemente umedecidos para evitar o ressecamento. Os pares de fêmures de cada animal foram divididos, aleatoriamente, em dois grupos. No primeiro grupo, foi instituído o teste de compressão axial (três pares). Após a fabricação de uma superfície de apoio em formato de semicírculo que se adequava à cabeça femoral (um ponto), aplicou-se carga sobre a mesma no sentido proximal para distal com o espécime na posição vertical com a base fixada em quatro pontos por parafusos, dentro de um cilindro metálico. No segundo teste, o fêmur foi posicionado horizontalmente e sua epífise distal com fixação similar ao teste anterior, realizou-se o teste de torção (em quatro pares de fêmures) aplicando-se força rotacional anti-horária por meio de três parafusos fixados em pontos equidistantes na sua epífise proximal. Ambos os testes foram executados em máquina universal de ensaios mecânicos (EMIC ${ }^{\circledR}$ DL10000) a velocidade de $20 \mathrm{~mm} / \mathrm{min}$ até ocorrer falha do implante ou fratura óssea, observando-se a carga máxima em $\mathrm{N} / \mathrm{mm}$ obtida durante o teste.

As análises estatísticas das variáveis: médias dos ângulos de Norberg e ICF de ambos os grupos (placa e HIB) foram comparadas entre os momentos T0 e T1 pelo teste test $t$ de student, bem como os dados dos ensaios biomecânicos (compressão e torção) e as médias dos tempos de execução de cada técnica. As avaliações foram efetuadas com significância de 95\% utilizando-se o software GraphPad Prism ${ }^{\circledR}$ versão 5.0.

\section{RESULTADOS}

Não houve diferença $(\mathrm{p}<0,05)$ na média do ângulo de Norberg entre T0 $\left(108,5 \pm 6,9^{\circ}\right)$ e $\mathrm{T} 1\left(112,08 \pm 8,65^{\circ}\right)$ no grupo placa. Entretanto, no grupo HIB, o ângulo apresentou aumento significativo $(\mathrm{p}<0,05)$ em T1 $\left(111,22^{\circ} \pm 3,89\right)$ quando comparada à T0 $\left(106,84 \pm 5,55^{\circ}\right)$.
Com relação ao ângulo de inclinação da cabeça e colo femoral (ICF), a média em T0 foi de $127,6 \pm 4,70^{\circ}$ no grupo placa e em T1 foi de $110,06 \pm 10,61^{\circ}$; no grupo HIB, a média em T0 foi de $126,43 \pm 5,87^{\circ}$ e em T1 de $116,87 \pm 8,62^{\circ}$. Ambos os grupos apresentaram diminuição do ângulo ICF após a cirurgia $(\mathrm{P}<0,05)$. Ao se comparar os valores de ICF após a cirurgia entre os grupos, não houve diferença entre as técnicas.

Não se observou diferença $(p>0,05)$ na avaliação do tempo dispendido para a implantação dos métodos de fixação grupo placa $(37 \pm 6,16$ minutos $)$ e o grupo $\operatorname{HIB}(38,2 \pm 6,9$ minutos).

Complicações na colocação dos implantes foram observadas em três fêmures (15\%) dos 20 operados. Destas, duas ocorreram no grupo HIB; em um dos casos, o segundo parafuso proximal ficou posicionado muito próximo à linha da osteotomia; e no outro, ocorreu erro no bloqueio do segundo parafuso proximal. Ambas as complicações só foram diagnosticadas na avaliação radiográfica pós-operatória. No grupo placa, houve frouxidão do parafuso mais proximal, observada no período trans-cirúrgico. Estes espécimes foram excluídos do ensaio biomecânico.

O ensaio biomecânico de compressão demonstrou que o grupo placa teve maior resistência $(863,3 \pm 74,46 \mathrm{~N} / \mathrm{mm})$ em comparação ao grupo $\operatorname{HIB}(586,7 \pm 44,10 \mathrm{~N} / \mathrm{mm})(\mathrm{P}=0,033)$. 0 ensaio de torção não apresentou diferença entre os grupos placa $(9,35 \pm 1,58 \mathrm{~N} / \mathrm{mm})$ e $\operatorname{HIB}(10,73 \pm 1,92 \mathrm{~N} / \mathrm{mm})$.

\section{DISCUSSÃO}

O propósito da osteotomia intertrocantérica varizante (OIV) é a redução do ângulo de inclinação da cabeça e colo femoral (ICF) e normoversão da cabeça femoral, permitindo melhor redistribuição das forças que atuam na cartilagem e osso acetabular de articulações displásicas (Moghadam et al. 2013). Após a realização do procedimento cirúrgico, embora ambos os grupos tenham demonstrado tendência de aumento na média do ângulo de Norberg, esta alteração foi significativa apenas no grupo HIB. Pinna et al. (2013), estudando os efeitos da OIV no ângulo de Norberg e porcentagem de recobrimento acetabular de cães displásicos, notaram expressiva diminuição das subluxações e melhora na congruência articular. Acredita-se que os dados relacionados ao ângulo de Norberg, no presente estudo, não tenham sido tão expressivos devido a OIV ter sido realizada em cadáveres não portadores de displasia.

Com relação ao ângulo de ICF, os valores encontrados em T0 foram considerados normais de acordo com Pinna et al. (2013) e Rumph \& Hathcock (1990). Houve diferença significativa na comparação entre os ângulos nos momentos T0 e T1 nos dois grupos estudados. Tais observações indicaram que, independente do método de fixação aplicado, o objetivo do procedimento foi atingido. Outros estudos obtiveram resultados semelhantes utilizando a OIV, estabilizada com placa compressiva, com o propósito de reduzir o ângulo de ICF como Evers et al. (1997) e Pinna et al. (2013).

Sabe-se que a placa compressiva pode ser moldada (Evers et al. 1997, Lins 2011) e, diante desta característica esperava-se que no grupo placa houvesse redução mais im- 
portante do ICF pós OIV em relação ao grupo HIB. Pois após realização da osteotomia em cunha e redução dos segmentos formados, há inclinação do segmento proximal, fazendo com que a fossa intertrocantérica não fique mais alinhada com o canal medular (Inoe et al. 1996). A HIB, por tratar-se de implante rígido, não moldável e aplicado no canal medular (Schmaedecke 2007), poderia ter impedido diminuição do ICF. Entretanto, é possível que a modificação do local de introdução da haste (através do trocanter maior ao invés da fossa intertrocantérica) e adequação da localização dos orifícios proximais destes implantes tenham permitido adequada angulação da cabeça/cólo femorais. Assim, os resultados do presente estudo não revelaram diferença entre os ângulos de inclinação com relação aos métodos de fixação. Infelizmente, não foram encontrados durante esta compilação, dados que pudessem ser confrontados com os resultados obtidos.

Com relação à agilidade na realização dos procedimentos, embora Duhautois (2003) e White et al. (2006) tenham citado como grande vantagem da utilização da HIB em relação às placas compressivas nas fraturas diafisárias, no presente estudo este fato não foi evidenciado. Acredita-se que a modificação do local de introdução da haste, localização muito proximal da osteotomia e pequena dimensão do segmento ósseo proximal tenham influenciado na dificuldade e complexidade da estabilização ósseas e, consequentemente, refletido no tempo de implantação da haste.

Como complicações do procedimento, houve erro de bloqueio no segundo parafuso proximal da haste em um dos cães, provavelmente devido a desvio iatrogênico da broca no momento da perfuração. Complicações semelhantes já foram relatadas por outros autores, todavia mais frequentes nos parafusos distais (Duhautois 2003, Piórek et al. 2012). Outra complicação relacionada à HIB foi à proximidade do segundo parafuso proximal ao foco de osteotomia. Esta situação foi resultado das limitadas dimensões do segmento proximal e da impossibilidade de angulação dos parafusos da haste para o devido bloqueio. Não foi encontrado na literatura este tipo de intercorrência, pois a HIB é mais comumente utilizada em fraturas diafisárias do que epifisárias (Moses et al. 2002, Duhautois 2003). No grupo placa, em um dos animais, houve frouxidão do parafuso mais proximal do implante. Goh et al. (2009) também citaram a ocorrência de afrouxamento de parafusos com o uso de placa, principalmente quando os parafusos são monocorticais, como foi o caso. 0 uso de parafusos monocorticais se fez necessário devido ao tamanho do segmento proximal, pois os mesmos são direcionados para a cabeça femoral e não devem invadir a articulação.

Embora a HIB tenha se mostrado anteriormente mais resistente que placas compressivas em fraturas diafisárias (Piórek et al. 2012) o mesmo não foi observado na estabilização da osteotomia epifisária no presente estudo. A placa compressiva proporcionou maior resistência biomecânica no ensaio de compressão e resultados semelhantes no ensaio de torção quando comparado à HIB. Há hipótese de que a diferença de diâmetro dos parafusos da placa compressiva $(3,5 \mathrm{~mm})$ e da HIB $(2,7 \mathrm{~mm})$ possam ter demostra- do esta maior resistência. Entretanto, é válido ressaltar que animais hígidos com peso corporal em torno de $30 \mathrm{~kg}$, apresentam valores máximos de carga vertical em plataforma de força de $315 \mathrm{~N}$ ao correr e trotar (Goh et al. 2009). Sendo assim, é possível que ambos os implantes sejam capazes de bloquear as forças atuantes na deambulação normal de um animal, pois a falha ocorreu com média de $863,3 \pm 74,46 \mathrm{~N} /$ $\mathrm{mm}$ na placa e $586,7 \pm 44,10 \mathrm{~N} / \mathrm{mm}$ na HIB. Entretanto, ensaio clínico com avaliação das cargas cíclicas ainda precisa ser efetuado para confirmar esta hipótese.

Finalmente, o emprego da técnica deve ser futuramente testado em cães portadores de displasia, para verificar se os resultados seguirão os mesmos padrões observados no presente estudo.

\section{CONCLUSÕES}

0 uso de hastes intramedulares bloqueadas (HIB) na estabilização pós-osteotomia intertrocantérica varizante (OIV) foi exequível em cadáveres caninos, obtendo-se resultados similares na redução do ângulo de inclinação da cabeça e colo femoral (ICF) e no tempo cirúrgico em relação às placas compressivas em cães hígidos.

A placa compressiva demonstrou maior resistência biomecânica no ensaio de compressão e resultados equivalentes ao ensaio de torção em relação à HIB.

Agradecimentos.- À Fundação de Amparo à Pesquisa do Estado do Rio Grande do Sul (FAPERGS), pela bolsa de mestrado.

\section{REFERÊNCIAS}

Ciarlini L.D.R.P., Júnior A.G.A., Muniz L.M.R., Louzada M.J.Q., Oliva V.N.L.S. \& Ciarlini P.C. 2009. Avaliação comparativa de diferentes métodos de mensuração radiográfica utilizados para o diagnóstico da displasia coxofemoral de cães. Vet. Zootec. 16:385-393.

Duhautois B. 2003. Use of veterinary interlocking nails for diaphyseal fractures in dogs and cats: 121 cases. Vet. Surg. 32:8-20.

Evers P., Kramek B.A., Wallace L.J., Johnston G.R. \& King V. 1997. Clinical and radiographic evaluation of intertrochanteric osteotomy in dogs: a retrospective study of 18 dogs. Vet. Surg. 26:217-222.

Goh C.S., Santoni B.G., Puttlitz C.M. \& Palmer R.H. 2009. Comparison of the mechanical behaviors of semicontoured, locking plate-rod fixation and anatomically contoured, conventional plate-rod fixation applied to experimentally induced gap fractures in canine femora. Am. J. Vet. Res. 70:23-29.

Inoe A.P., Sampaio R.L., Machado V.M.V. \& Cruz M.L. 1996. Osteotomia femoral em cúpula para correção do ângulo de inclinação do colo do fêmur. Ciência Rural 6:153-157.

Lins B.T. 2011. Avaliação biomecânica comparativa de duas técnicas de osteotomia varizante proximal do fêmur: estudo em cadáveres de cães. Tese de Doutorado, Faculdade de Medicina Veterinária e Zootecnia, Universidade de São Paulo, São Paulo. Disponível em <http://www.teses. usp.br/teses/disponiveis/5/5140/tde-04052011-173111/en.php> Acesso em 25 ago. 2013.

Moghadam M.H., Moradi A. \& Omidi-Kashani F. 2013. Clinical outcome of femoral osteotomy in patients with legg-calvé-perthes disease. Arch. Bone Joint Surg. 1:90-93.

Moses P.A., Lewis D.D., Lanz O.I., Stubbs W.P., Cross A.R. \& Smith K.R. 2002. Intramedullary interlocking nail stabilisation of 21 humeral fractures in 19 dogs and one cat. Aust. Vet. J. 80:336-343.

Pinna S., Pizzuti E. \& Carli F. 2013. Effects of intertrochanteric varus osteotomy on Norberg angle and percent coverage of the femoral head in displastic dogs. J. Vet. Sci. 14:185-191.

Piórek A., Adamiak Z., Matyjasik H. \& Zhalniarovich Y. 2012. Stabilization 
of fractures with the use of veterinary interlocking nails. Pak. Vet. J. 32:10-14.

Prieur W.D. 2005. Osteotomia intertrocantérica. In: Bojrab M.J. (Ed.), Técnicas Atuais em Cirurgia de Pequenos Animais. $3^{a}$ ed. Roca, São Paulo. 920p.

Rumph P.F. \& Hathcock J.T. 1990. A symmetric axis-based method for measuring the projected femoral angle of inclination in dogs. Vet. Surg. 19:328-333.

Schmaedecke A. 2007. Avaliação biomecânica de diferentes bloqueios transcorticais de interlocking nail em relação às forças de torsão, encurvamento e axiais atuantes em fraturas diafisárias de fêmur de cães: estudo in vitro. Tese de Doutorado, Faculdade de Medicina Veterinária e Zootecnia, Universidade de São Paulo, São Paulo. Disponível em <http:// www.teses.usp.br/teses/disponiveis/10/10137/tde-06062007154221/pt-br.php> Acesso em 27 ago. 2013.

Vieira G.T., Tôrres R.C., Barros G.S., Rocha B.D. \& Rezende C.M. 2010. Associação entre o ângulo de Norberg, o percentual de cobertura da cabeça femoral, o índice cortical e o ângulo de inclinação em cães com displasia coxofemoral. Arq. Bras. Med. Vet. Zootec. 62:1094-1101.

White T.O., Clutton R.E., Salter D., Swann D., Christie J. \& Robinson C.M. 2006. The early response to major trauma and intramedullary nailing. Bone Joint Surg. 88:823-827. 